\title{
Aristida helleriana (Poaceae, Aristidoideae), una Nueva Especie Endémica del Pampa en Rio Grande do Sul, Brasil
}

Author(s): Marene Machado MarchiJaime Mujica SallésRosa Lía Barbieri

Source: Novon: A Journal for Botanical Nomenclature, 24(3):261-265.

Published By: Missouri Botanical Garden

DOI: http://dx.doi.org/10.3417/2013028

URL: http://www.bioone.org/doi/full/10.3417/2013028

BioOne (www.bioone.org) is a nonprofit, online aggregation of core research in the biological, ecological, and environmental sciences. BioOne provides a sustainable online platform for over 170 journals and books published by nonprofit societies, associations, museums, institutions, and presses.

Your use of this PDF, the BioOne Web site, and all posted and associated content indicates your acceptance of BioOne's Terms of Use, available at www.bioone.org/ page/terms_of_use.

Usage of BioOne content is strictly limited to personal, educational, and noncommercial use. Commercial inquiries or rights and permissions requests should be directed to the individual publisher as copyright holder. 


\title{
Aristida helleriana (Poaceae, Aristidoideae), una Nueva Especie Endémica del Pampa en Rio Grande do Sul, Brasil
}

\author{
Marene Machado Marchi \\ Postdoctora, Embrapa Clima Temperado, Becaria de la Coordenação de Aperfeiçoamento de \\ Pessoal de Nível Superior (CAPES), Av. Alberto Rosa, 155/apt. 202, Centro, Pelotas, Rio Grande \\ do Sul, CEP 96010-770, Brasil. marene.marchi@gmail.com

\section{Jaime Mujica Sallés} \\ Docente del Departamento de Antropología e Arqueología de la Universidade Federal de Pelotas, \\ Brasil.mujica.jaime@gmail.com

\section{Rosa Lía Barbieri} \\ Docente del Programa de Postgrado en Agronomía/Fitomejoramiento, Faculdade de Agronomia \\ Eliseu Maciel, Universidade Federal de Pelotas, Investigadora de la Empresa Brasileira de \\ Pesquisa Agropecuaria/Embrapa Clima Temperado, Brasil. lia.barbieri@embrapa.br
}

Resumen. Aristida helleriana M. Marchi, J. Mujica \& R. L. Barbieri es una especie nueva de Poaceae (Aristidoideae) para la ciencia colectada en la Estancia São Miguel, en Tapes, Rio Grande do Sul (Brasil), durante el estudio florístico del área de conservación in situ de Butia odorata (Barb. Rodr.) Noblick (Arecaceae). Aristida helleriana se diferencia de A. laevis (Nees) Kunth, la especie más afín y también presente en el área de estudio, por poseer panícula pauciflora, contraída, con ramas con espiguillas dispuestas en forma helicoidal y discontinuas a lo largo del raquis, dejándolo parcialmente visible en algunos tramos. La nueva especie se distingue por las lemas menores, 1-3 mm (vs. 8.5-13 mm en A. laevis) y con aristas más cortas, 11-17(19) mm (25-50 mm en A. laevis).

Abstract. Aristida helleriana M. Marchi, J. Mujica \& R. L. Barbieri is a new species of Poaceae (Aristidoideae) collected at São Miguel's Farm, in Tapes, Rio Grande do Sul State (Brazil), during a floristic survey in an area where in situ conservation of Butia odorata (Barb. Rodr.) Noblick (Arecaceae) has been done. Aristida helleriana differs from A. laevis (Nees) Kunth, its closely related species in the study area, due to its few-flowered panicle, with branches bearing spikelets arranged helically and discontinuously along the rachis, leaving it fully exposed in some sections. The new species is distinguished by its lower lemmas 1-3 $\mathrm{mm}$ (vs. 8.5-13 $\mathrm{mm}$ in A. laevis) and shorter awns 1117(19) $\mathrm{mm}$ (25-50 $\mathrm{mm}$ in A. laevis).

Key words: Aristida, Aristidoideae, Brazil, Butia, IUCN Red List, pampa biome, Poaceae, Rio Grande do Sul.
El área de conservación in situ de la palmera Butia odorata (Barb. Rodr.) Noblick (Arecaceae) en la Estancia São Miguel, en Tapes, forma parte del bioma pampa, en Rio Grande do Sul, Brasil. La región comprende 750 hectáreas de vegetación de campo nativo y posee la mayor concentración de esta especie de palmera en el país. Es un área conservada por sus propietarios desde ya hace más de cien años. Desde 2010 existe un convenio entre sus dueños y la Empresa Brasileira de Pesquisa Agropecuária-Embrapa Clima Temperado, para estudiar el área y sentar las bases para su manejo y uso sustentable. De esta forma, hoy comprende el área de conservación del Banco de Germoplasma in situ de B. odorata. Longhi-Wagner (2015) cita 16 especies de Aristida L. para el bioma pampa, 37 para todo Brasil, con 11 endémicas. De acuerdo con el relevamiento realizado por los autores en el área de estudio, fueron constatadas ocho especies de este género, una de las cuales, nueva para la ciencia, se describe en este trabajo.

Aristida es un género cosmopolita, de regiones tropicales y subtropicales, con 304 especies (Stevens, 2001). Estudios taxonómicos fueron realizados por diversos autores a lo largo del siglo XX. La mayor revisión del género fue hecha por Henrard (1926, 1932, 1933); posteriormente Allred (2003) trató el género para América del Norte; Valdés y Allred (2003) lo trataron para Mexico; Pohl y Davidse (1994) en la Flora Mesoamericana; Caro (1969, 1970), Nicora (1978), Nicora y Rúgolo de Agrasar (1987) y Sulekic $(2003,2012)$ lo estudiaron para Argentina; Lombardo y Rosengurtt (1984) y Rosengurtt et al. (1970) para Uruguay. En Brasil se han realizado 
diversos trabajos en Aristida, entre ellos: LonghiWagner (1990, 1992, 1999), Longhi-Wagner et al. (2001), Renvoize (1984, 1988) y Smith et al. (1982).

Aristida helleriana M. Marchi, J. Mujica \& R. L. Barbieri, sp. nov. TIPO: Brasil. Rio Grande do Sul: Tapes, Estancia São Miguel, área de conservación del Banco de Germoplasma in situ de Butia odorata, $30^{\circ} 31^{\prime} 46,11^{\prime \prime} \mathrm{S}$ $51^{\circ} 21^{\prime} 47,61^{\prime \prime} \mathrm{W}, 50 \mathrm{~m}, 11$ Nov. 2012, M. M. Marchi \& J. Mujica 3991 (holotipo, ICN; isotipos, Herbário da Embrapa Clima Temperado [HECT], MO). Figura 1.

Diagnosis. Similar to Aristida laevis (Nees) Kunth in being a robust perennial plant, with glabrous sheaths, a pubescent collar, and the erect, contracted inflorescences, but distinguished from it by the helicoid and discontinuous arrangement of the inflorescence branches that expose the inflorescence axis in some sections and the lower lemmas 1-3 mm (vs. 8.5-13 $\mathrm{mm}$ in A. laevis) with shorter awns 1117(19) $\mathrm{mm}$ (vs. 25-50 mm).

Planta perenne, erecta, rizomatosa, formando matas robustas, con inflorescencias exsertas y erectas, de 70-110 cm de altura. Vainas foliares glabras, más largas que los entrenudos, lóbulos con pelos blancos, de 1-3 mm de largo, esparcidos, o glabros en las hojas secas; cuello marcado con línea de tricomas generalmente continua sobre la nervadura central (salvo en las hojas más viejas), perceptible al tacto, y ocasionalmente tricomas hialinos de $1 \mathrm{~mm}$ de largo; lígula poco visible, truncada, cortamente ciliada, de $0.2 \mathrm{~mm}$ de largo; láminas foliares flexuosas o erectas, escabriúsculas superiormente en los márgenes, glabras, conduplicadas o convolutas de (17)28-48(77) $\times(0.1) 0.22$ $0.4(0.5) \mathrm{cm}$, con diminutas asperezas antrorsas sobre las nervaduras en ambas caras; las más viejas, secas, planas y recurvadas superiormente; ápice navicular. Inflorescencia erecta, pauciflora, contraída, gris-violácea, con ramas de espiguillas dispuestas de forma helicoidal y discontinuas a lo largo del raquis, dejando este expuesto en algunos tramos, de (5)10-28 $\times(0.5) 1.2-2 \mathrm{~cm}$ de largo, incluyendo las aristas. Espiguillas con glumas lanceoladas, aristadas, 1-nervadas; glumas inferiores menores que las superiores, de (5) $5.2-7(7.5) \times$ 0.6-0.8(1.3) $\mathrm{mm}$ de largo, violáceas o paleáceas, glabras, con asperezas antrorsas en el dorso de la nervadura central, aristas de $1.5-3.5(4) \times 0.6-0.8$ $\mathrm{mm}$; glumas superiores de 6.6-9.5(11) $\times 0.3-$ 0.8(1.3) mm, aristas de (1.6)1.8-2.8(3.5) mm; lema cilíndrica, con los márgenes convolutos, sin surco, triaristada, sin columna, de $1-3 \times 0.5-0.6 \mathrm{~mm}$, callo de 0.6-0.65 mm de largo, agudo, glabro; aristas verdes, subiguales, la central algo mayor, con asperezas antrorsas, la central de 12.8-17(19) $\mathrm{mm}$ de largo y las laterales de $11-16.2 \mathrm{~mm}$ de largo; pálea hialina, aguda, de 3.8-4 mm de largo; lodículas 2, hialinas, $0.4 \mathrm{~mm}$ de largo; anteras 3 , de 0.4-0.6 mm de largo; ovario poco desarrollado, de $0.4 \mathrm{~mm}$ de largo. Cariopsis no observada.

Fenología. Aristida helleriana florece desde octubre hasta enero. Aún no se han observado cariopsis de la especie.

Distribución y hábitat. Esta especie fue colectada por primera vez en el local de monitoreo denominado Área 1, en la Estancia São Miguel, en Tapes, Rio Grande do Sul, Brasil, en 2010, durante el relevamiento florístico realizado por la primera autora como parte de su tesis de Doctorado. También fue colectada en las áreas 2 y 3 de monitoreo permanente. Las referidas áreas son mantenidas como clausuras, es decir, excluidas del pastoreo y el surgimiento de las especies vegetales está siendo acompañado desde el año 2010.

Categoría de la Lista Roja de la IUCN. La población de Aristida helleriana está bien representada en esta área particular protegida de 750 hectáreas, en el bioma pampa. Se encuentra tanto dentro de las tres áreas de estudio, como fuera de las mismas, mas siempre asociada a la palmera Butia odorata. Como todos los hábitats de palmeras nativas de Rio Grande do Sul, su área de ocurrencia está amenazada, principalmente por la expansión urbana y la implantación de monocultivos (silvicultura, arroz y soja). Por estas razones A. helleriana es considerada VU o vulnerable, Al (c,e), de acuerdo con los criterios de la IUCN (2001).

Etimología. Aristida helleriana recibió esta denominación en homenaje a la Señora Nair Heller de Barros, propietaria de la Estancia São Miguel y responsable por la conservación de un remanente de Butia odorata en Brasil, donde la especie fue encontrada.

Discusión taxonómica. Aristida helleriana es similar a A. laevis (Nees) Kunth, siendo una planta robusta, perenne, con hojas erectas desde la base y ápices flexuosos, con el cuello de la vaina marcado con una línea continua de tricomas y con una panícula contraída, linear y erecta. Se diferencia fácilmente de A. laevis y se identifica en campo, por su característica panícula apical con ramas de espiguillas dispuestas en forma helicoidal y discontinuas a lo largo del raquis, el cual queda parcialmente 


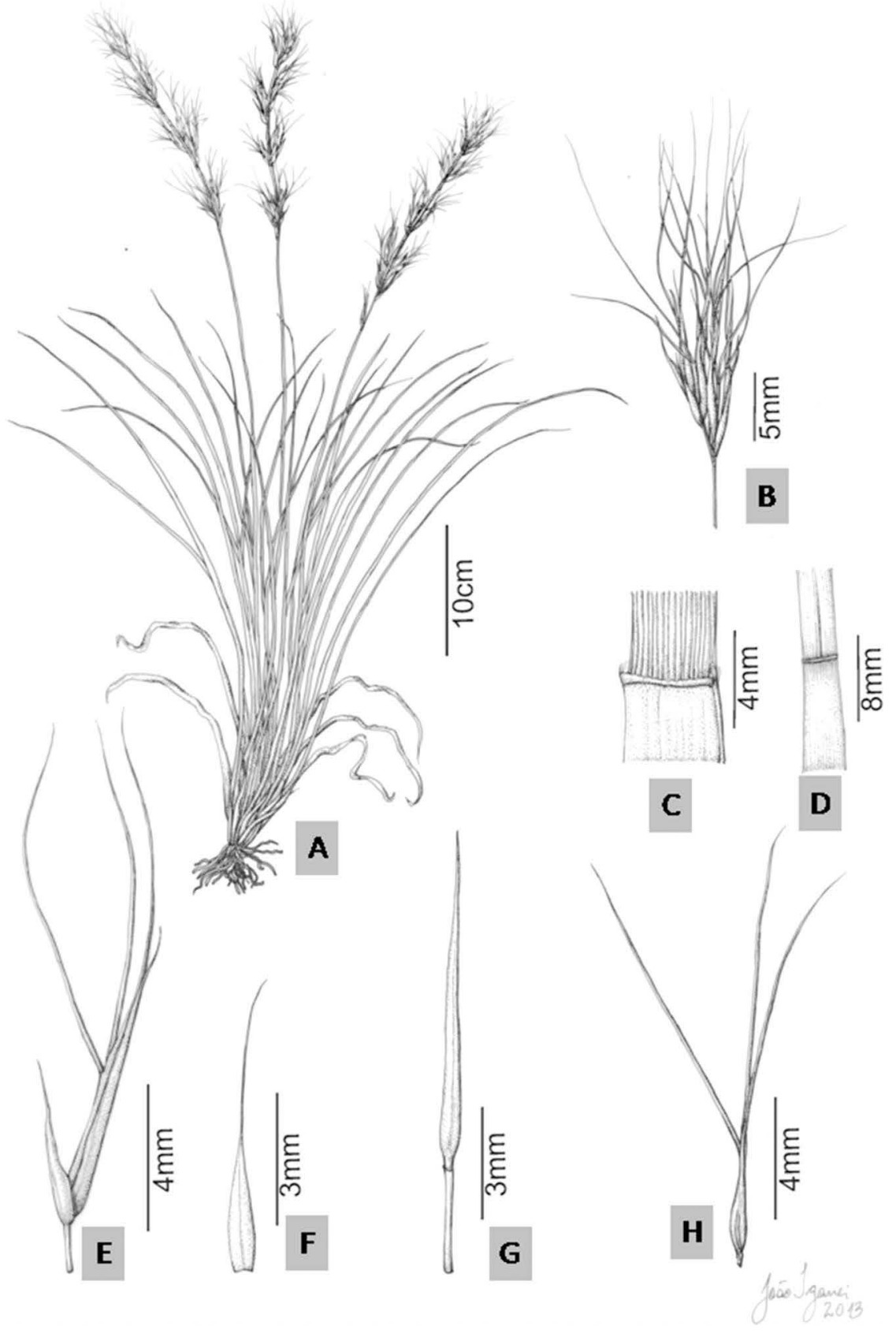

Figura 1. Aristida helleriana M. Marchi, J. Mujica \& R. L. Barbieri. —A. Hábito fértil. —B. Rama con espiguillas. —C. Lígula truncada, cortamente ciliada y lóbulos con pelos. - D. Cuello marcado con línea de tricomas continua sobre la nervadura central. -E. Espiguilla, vista lateral. —F. Gluma inferior, vista dorsal. —G. Gluma superior, vista dorsal. —H. Lema mostrando callo agudo. Dibujo del holotipo M. M. Marchi \& J. Mujica 3991 (ICN).

desnudo en algunos tramos. Otra característica fundamental para confirmar la identificación de $A$. helleriana, que puede ser observada en el laboratorio, es el pequeño tamaño de la lema y de las aristas, que miden respectivamente, de 1-3 mm de largo y 11-
17(19) $\mathrm{mm}$ de largo, mientras que en el material colectado en la misma área, de A. laevis, la lema y las aristas miden de 9.5-12.5 mm de largo y 25-48 mm de largo, respectivamente. De acuerdo con los datos proporcionados por la Lista de Espécies da Flora do 
Brasil (2015), el género Aristida está representado por 37 especies en el país, con 16 especies ocurriendo en el bioma pampa. De estos taxones citados para la pampa brasilera, siete (A. circinalis Lindm., A. flaccida Trin. \& Rupr., A. jubata (Arechav.) Herter, A. laevis, A. megapotamica Spreng. var. megapotamica, A. riograndensis Severo \& Boldrini y A. venustula Arechav. var. venustuloides (Caro) Longhi-Wagner) se encuentran en el área de la estancia, además de la especie nueva. Entre estas vale destacar la existencia, en las áreas de estudio, de dos especies endémicas de Brasil, A. riograndensis y A. flaccida, que junto con A. helleriana, refuerzan la importancia del área de conservación in situ de Butia odorata.

Clave para Diferenciar Aristida helleriana de las Siete Especies de Aristida que se Encuentran en el Ecosistema de Palmar en Tapes, Rio Grande do Sul, Brasil

1a. Aristas laterales de la lema menores que la arista central ............................. 2

2a. Panícula espiciforme. Lema con columna retorcida de 50-53 mm de largo .............. ...... A. megapotamica Spreng. var. megapotamica

2b. Panículas no espiciformes. Lema sin columna ..... 3

3a. Panícula laxa, pauciflora .. A. flaccida Trin. \& Rupr.

3b. Panícula contraída, linear ...... A. circinalis Lindm.

1b. Aristas de la lema subiguales ............. 4

4a. Cuello de la vaina con una línea de tricomas cortos ............................ 5

5a. Panícula abierta a subcontraída, nutante. Lema con aristas de 60-100 mm de largo ......... ............ A. riograndensis Severo \& Boldrini

5b. Panícula contraída, linear, erecta. Lema con aristas de 11-48 mm de largo .............6 6

6a. Panícula apical continua. Lema con 9.5-12.5 mm de largo, aristas con 25-48 mm de largo ....... A. laevis (Nees) Kunth

6b. Panícula apical con ramas discontinuas, exponiendo el raquis. Lema con $1-3 \mathrm{~mm}$ de largo, aristas con 11-17(19) mm de largo ..........

A. helleriana M. Marchi, J. Mujica \& R. L. Barbieri

4b. Cuello de la vaina glabro $\ldots \ldots \ldots \ldots \ldots \ldots \ldots .7$

7a. Panícula nutante. Lema con apéndice hialino apical 1-2 mm de largo, aristas (110)160200(225) mm de largo .. A. jubata (Arechav.) Herter

7b. Panícula erecta. Lema sin apéndice hialino apical, aristas con 60-80(100) mm de largo ..

.... A. venustula Arechav. var. venustuloides (Caro)

Longhi-Wagner

Paratipos. BRASIL. Rio Grande do Sul: Tapes, Est. São Miguel, área de conservación del Banco de Germoplasma in situ de Butia odorata, 30 $31^{\prime} 22,34^{\prime \prime} \mathrm{S}$ $51^{\circ} 21^{\prime} 35,23^{\prime \prime} \mathrm{W}$ (área de monitor. 1), $40 \mathrm{~m}, 4$ nov. 2010, M. M. Marchi \& J. Mujica 2709 (ICN), 30 $32^{\prime} 43,79^{\prime \prime} \mathrm{S}$ $51^{\circ} 22^{\prime} 6,35^{\prime \prime} \mathrm{W}$ (área de monitor. 3), $40 \mathrm{~m}, 7$ dic. 2010, $M$. M. Marchi \& J. Mujica 2739, 2740 (Herbário da Embrapa Clima Temperado [HECT], MO); $30^{\circ} 32^{\prime} 43,79^{\prime \prime} \mathrm{S}$ $51^{\circ} 22^{\prime} 6,35^{\prime \prime} \mathrm{W}$ (área de monitor. 3), 40-50 m, 1 nov. 2012, M. M. Marchi \& J. Mujica 3934 (Herbário da Embrapa Clima Temperado [HECT], MO, PEL).
Agradecimientos. A la Estancia São Miguel, al Probio II, a Fapergs, al Conselho Nacional de Desenvolvimento Científico e Tecnológico (CNPq), al RS Biodiversidade por el apoyo y financiamiento del trabajo, y al Dr. João Iganci por la realización de la ilustración botánica.

\section{Literatura Citada}

Allred, K. 2003. Aristida L. Pp. 315-342 in M. E. Barkworth, K. M. Capels, S. Long \& M. B. Piep (editors), Flora of North America, Vol. 25. Magnoliophyta: Commelinidae (in part), Poaceae, Pt. 2. Oxford University Press, New York.

Caro, J. A. 1969. Aristida L. Pp. 208-224 en A. Burkart (editor), Flora Ilustrada de Entre Ríos (Argentina), Gramíneas, Vol. 6(2). INTA, Buenos Aires.

Caro, J. A. 1970. Aristida L. Pp. 352-369 en A. L. Cabrera (editor), Flora de la Provincia de Buenos Aires, Vol. 4(2). Gramíneas. INTA, Buenos Aires.

Henrard, J. T. 1926. A critical revision of the genus Aristida. Meded. Rijks-Herb. 54: 1-220.

Henrard, J. T. 1932. A monograph of the genus Aristida. Meded. Rijks-Herb. 2(58): 157-325.

Henrard, J. T. 1933. A critical revision of the genus Aristida. Meded. Rijks-Herb. 54(C): 703-747.

IUCN. 2001. IUCN Red List Categories and Criteria, Version 3.1. Prepared by the IUCN Species Survival Commission. IUCN, Gland, Switzerland, and Cambridge, United Kingdom. <http://www.iucn.org >, consultada 6 mayo 2014.

Lista de Espécies da Flora do Brasil. 2015. O gênero Aristida. <http://floradobrasil.jbrj.gov.br/>, consultada 8 julio 2014.

Lombardo, A. \& B. Rosengurtt. 1984. Aristida L. Pp. 157165 en Flora Montevidensis, t. 3. Gramíneas. Inst. Mun. Montevideo, Montevideo.

Longhi-Wagner, H. M. 1990. Diversidade e distribuição geográfica das espécies de Aristida L. (Gramineae) ocorrentes no Brasil. Acta Bot. Brasil. 4(1): 105-124.

Longhi-Wagner, H. M. 1992. Two new species of Aristida (Poaceae) from Brazil. Novon 2: 36-40.

Longhi-Wagner, H. M. 1999. O gênero Aristida (Poaceae) no Brasil. Bol. Inst. Bot. (São Paulo) 12: 113-179.

Longhi-Wagner, H. M. 2015. Aristida in Lista de Espécies da Flora do Brasil. Jardim Botânico do Rio de Janeiro, Rio de Janeiro. <http://floradobrasil.jbrj.gov.br/jabot/ floradobrasil/FB12989>, consultada 10 septiembre 2015.

Longhi-Wagner, H. M., V. Bittrich, M. G. L. Wanderley \& G. J. Shepherd (editoras). 2001. Aristida L. Pp. 10-17 en M. G. L. Wanderley, G. J. Shepherd \& A. M. Giulietti (coordenadores), Flora Fanerogâmica do Estado de São Paulo, Vol. 1. Hucitec, São Paulo.

Nicora, E. G. 1978. Aristida L. en M. N. Correa (editora), Flora Patagónica. Colecc. Ci. Inst. Nac. Tecnol. Agropecu. 8 (3): 472-478.

Nicora, E. G. \& Z. E. Rúgolo de Agrasar. 1987. Aristida L. Pp. 333-335 en E. G. Nicora \& Z. E. Rúgolo de Agrasar, Los Géneros de Gramíneas de América Austral. Hemisferio Sur, Buenos Aires.

Pohl, R. W. \& G. Davidse. 1994. Aristida L. Pp. 253-257 en G. Davidse, M. Souza S. \& A. O. Chater (editores), Flora Mesoamericana, Vol. 6. Alismataceae-Cyperaceae. 
Universidad Nacional Autónoma de México, D.F., México City, and Missouri Botanical Garden, St. Louis.

Renvoize, S. A. 1984. The Grasses of Bahia. Royal Botanic Gardens, Kew.

Renvoize, S. A. 1988. Hatschbach's Paraná Grasses. Royal Botanic Gardens, Kew.

Rosengurtt, B., B. R. Arrillaga de Maffei \& P. Izaguirre de Artucio. 1970. Gramíneas Uruguayas. Universidad de la República, Montevideo.

Smith, L. B., D. C. Wasshausen \& R. M. Klein. 1982. Gramíneas. Pp. 516-538 en P. R. Reitz (editor), Flora Ilustrada Catarinense. Herbário Barbosa Rodrigues, Itajaí.
Stevens, P. F. (2001 en adelante). Angiosperm Phylogeny Website. Version 12, julio 2012. < http://www.mobot.org/ MOBOT/research/APweb/>, consultada 13 agosto 2015. Sulekic, A. A. 2003. Revisión de las especies del género Aristida (Poaceae, Aristideae) del Noroeste de la Argentina. Darwiniana 41(1-4): 155-188.

Sulekic, A. A. 2012. Aristida L. Pp. 24-46 en F. O. Zuloaga, Z. E. Rugolo \& A. M. Anton (editores), Flora Argentina, Vol. 3(I). Monocotyledoneae. Poaceae: Aristidoideae a Pharoideae. Gráficamente Ediciones. Córdoba, Argentina.

Valdés R., J. \& K. W. Allred. 2003. El género Aristida (Gramineae) en el nordeste de México. Acta Bot. Mex. 63: $1-45$. 